\title{
Changes in eating behavior through lifestyle treatment in women with polycystic ovary syndrome (PCOS): a randomized controlled trial
}

Geranne Jiskoot ( $\sim$ L.jiskoot@erasmusmc.nl )

Erasmus MC

Alexandra Dietz de Loos

Erasmus MC

Reinier Timman

Erasmus MC

Annemerle Beerthuizen

Erasmus MC

Joop Laven

Erasmus MC

Jan Busschbach

Erasmus MC

Research Article

Keywords:

Posted Date: February 7th, 2022

DOI: https://doi.org/10.21203/rs.3.rs-1323480/v1

License: (1) This work is licensed under a Creative Commons Attribution 4.0 International License.

Read Full License 


\section{Abstract}

\section{Background}

Eating behaviors like emotional eating, external eating and restrained eating play an important role in weight gain and weight loss in the general population. This has not yet been studied in women with Polycystic Ovary Syndrome (PCOS).

\section{Methods}

Women diagnosed with PCOS ( $\mathrm{N}=183$ ), with a body mass index $(\mathrm{BMI})>25 \mathrm{~kg} / \mathrm{m}^{2}$ and trying to achieve a pregnancy were either assigned to one year of 20 group sessions of cognitive behavioral therapy (CBT) combined with nutritional advice and exercise with additional 9 months of electronically tailored feedback through SMS (LS with SMS) or 20 group sessions of CBT combined with nutritional advice and exercise without SMS (LS without SMS), or CAU, which includes the advice to lose weight using publicly available services. Disordered eating was assessed with the Eating Disorder Examination Questionnaire (EDEQ).

Results

EDEQ scores worsened in CAU (+47.5\%) and improved in the lifestyle group (LS) (-4.2\%) at 12 months. The difference between the LS and CAU was significant $(p=0.007)$ and resulted in a medium to large effect size (Cohen's d: -0.72). No significant difference were observed in EDEQ scores between LS with SMS compared to LS without SMS (Cohen's d: $0.28 ; P=0.399$ ). Also, weight loss did not mediate the changes in eating behavior. An overall completion rate of 67/183 (36.6\%) was observed.

\section{Conclusions}

A three-component CBT lifestyle program resulted in significant improvements in disordered eating behavior compared to CAU. Changes in disordered eating behavior are important for long-term weight loss and mental health.

Trial registration: NTR, NTR2450. Registered 2 August 2010, https://www.trialregister.nl/trial/2344

\section{Summary}

Polycystic Ovary Syndrome (PCOS) is a disorder that affects many women (8-13\%). Many women with PCOS have a higher risk to develop an eating disorder like bulimia nervosa. Besides the higher risk for eating disorders, many women with PCOS have disordered eating behavior like emotional eating, restricting themselves from food and episodes of binge eating. We have examined if lifestyle treatment including cognitive behavioral therapy can help for disordered eating behavior. We compared a group of women in the lifestyle intervention to a group of women who had to lose weight by themselves. We found that especially the women in this group developed more disordered eating. While eating behaviour 
improved in the women of the lifestyle intervention. We believe these changes are important for long-term weight loss and mental health.

\section{Background}

Polycystic Ovary Syndrome (PCOS) is a common endocrine disorder that affects $8-13 \%$ of women in their reproductive years (1-3). The prevalence of overweight and obesity is significantly higher in women diagnosed with PCOS compared to women without PCOS $(4,5)$. Most women with PCOS have overweight or obesity throughout their entire lifespan $(6,7)$. Besides obesity, many women with PCOS experience depressive and anxiety complaints, have lower self-esteem and experience a more negative body-image compared to women without PCOS (8-10). Other psychological aspects such as a lower quality of life and disordered eating have a major impact on women with PCOS (11).

Feeding and eating disorders, such as anorexia nervosa, bulimia nervosa and binge eating disorder (BED) are diagnosed according to the $5^{\text {th }}$ version of the Diagnostic and Statistical Manual of Mental Disorders (DSM-5) (12). Besides these official eating disorders, many individuals do not fulfill all the criteria of an eating disorder while having disordered eating patterns (13). Disordered eating includes the full spectrum of eating-related problems like emotional eating, restrained eating and episodes of binge eating (14). There are three psychological theories of eating behavior described in the literature: the psychosomatic theory, the externality theory and the restraint theory. The Dutch Eating Behavior Questionnaire (DEBQ) was developed to measure these three eating behaviors: emotional eating, external eating and dietary restraint (15). Emotional eating is defined as eating in response of stress or negative emotions (16) and is associated with overweight and weight gain (17). Following a strict diet is considered a risk factor for emotional eating (18). External eating is defined as overeating in response to the sight and smell of attractive food (19). External eating is associated with a higher body mass index (BMI) and overweight. Emotional eating tends to co-occur with external eating (19). Restrained eating refers to "chronic dieting" or intentional restriction of food intake to influence body weight, often interrupted with episodes of overeating (or eating more than wanted). After these periods of overeating or eating "forbidden" foods, restraint eaters tend to consume more in general $(20,21)$.

Most treatments for obesity are designed to increase dietary restraint. The problem of these restricted diets is that participants regain most of the lost body weight after stopping their diet. In fact; restricted diets work counterproductive; with some patients even ending up weighing more than before the diet (22). Restricted diets are also a significant contributor to binge eating (23). Therefore, the treatment of people who have obesity should be tailored based on the type of eating behavior and focus on emotion regulation skills to achieve long term weight loss (19). Cognitive behavioral therapy (CBT) seems the best solution for individuals who have obesity to develop healthy eating behavior and to prevent relapse (24). Also, CBT has proven to be effective for individuals with bulimia nervosa and binge eating disorder (25).

In women with PCOS, the odds for bulimia nervosa (OR 1.37), binge eating (OR 2.95) and any eating disorder (OR 1.96) are higher than in the general population (26). Binge eating symptoms were more 
often present in women with PCOS compared to healthy controls (27). Besides the increased odds for eating disorders, many women with PCOS have disordered eating behavior like emotional eating, dietary restraint and episodes of binge eating. The odds for disordered eating were three times higher in women with PCOS compared to control (28). Also, women with PCOS score higher on the Eating Disorder Examination Questionnaire (EDEQ) than women in the general population (28). Especially the group of women with PCOS who also have obesity or high depression scores seems at risk for disordered eating (29). Contrary to these results, Larsson and colleagues found no significant differences for restrained eating, uncontrolled eating, or emotional eating between women with PCOS and women without PCOS. However, women with PCOS did have higher scores on the Eating Attitudes Test compared with women without PCOS before and after adjustment for age and BMI (30). This suggests that women with PCOS do struggle more with weight loss attempts and weight control than women in the general population.

Weight loss by a three-component lifestyle intervention is recommended as first-line treatment for women with PCOS (31). Compared to one or two-component lifestyle interventions, three-component lifestyle interventions have the biggest effect to establish a long-term weight loss in general (32). These threecomponent lifestyle interventions should consist of: development of a healthy diet in combination with exercise and cognitive behavioral therapy (CBT). Important principles and techniques of the CBT component are self-monitoring, realistic and achievable goal setting, control of dangerous stimuli and triggers and promotion of alternative behaviors during critical emotional situations or negative mood states (33). CBT is used in obesity treatment as a technique for challenging and changing dysfunctional eating and body-related beliefs and schemas to develop and maintain a healthier eating pattern (24).

A lifestyle intervention (LS) was designed to examine the effectiveness of a 1-year three-component multidisciplinary program with or without Short Message Service (SMS) for women with PCOS and a BMI above 25 (34). The mean weight loss was $2.32 \mathrm{~kg}$ in care as usual (CAU), $4.65 \mathrm{~kg}$ in lifestyle without SMS (LS without SMS) and $7.87 \mathrm{~kg}$ in lifestyle with SMS (LS with SMS). More weight loss was observed in LS compared to CAU $(P<0.001)$ and even more in LS with SMS compared to LS without SMS $(P=0.017)(35)$. The current LS was designed to change behavior and achieve weight loss through this heathier lifestyle. Therefore, three hypotheses will be tested in this analysis: 1) a three-component LS (with or without SMS) is more effective than CAU for improving disordered eating behavior, 2) LS with SMS is more effective than LS without SMS and 3) androgens, weight and depression mediate the effects of LS on disordered eating behavior.

\section{Material And Methods}

\section{Study design}

We performed a longitudinal RCT measuring the effectiveness of a three-component multidisciplinary 1year LS in women with PCOS and overweight or obesity. This study was approved by the Medical Research Ethics Committee of the Erasmus MC in Rotterdam; reference number MEC 2008-337 and registered at the Dutch Trial registration: reference number NTR2450. The current study on eating 
behavior represents an analysis of a secondary outcome. The results of the primary outcome and the design of the intervention have been described previously $(34,35)$.

\section{Participants}

We conducted this randomized controlled trial at the Division of Reproductive Endocrinology and Infertility, Department of Obstetrics and Gynecology of the Erasmus MC, Rotterdam, the Netherlands. Women were eligible if they were diagnosed with PCOS according to the Rotterdam 2003 consensus criteria, had a BMl above $25 \mathrm{~kg} / \mathrm{m}^{2}$, between 18 and 38 years old and would like to become pregnant. Women with inadequate command of the Dutch language, severe mental illness, obesity with another somatic cause, ovarian tumors that lead to an androgen excess, adrenal diseases, had other malformations of their internal genitalia or who were pregnant, were not eligible for the study.

At baseline, and at 3-, 6-, 9- and 12-months all participants attended the outpatient clinic for standardized screening and all outcome measures were assessed. This screening included a family and reproductive history, anthropomorphometric and ultra-sonographic assessments. Participants also completed the DEBQ, EDEQ and BDI-II questionnaires at all these time points.

\section{Lifestyle intervention (LS)}

The lifestyle treatment aimed at 1) changing cognitions by cognitive behavioral therapy (CBT); 2) developing healthy dietary habits; 3 ) encouraging and promoting physical daily activity, and; 4) activating social support. The intervention consisted of 20 group sessions of 2.5 hours carried out by a multidisciplinary team. The first 1.5 hours of every group session was supervised by a basic psychologist/CBT trainer and a dietician. The last hour of each session was supervised by two physical therapists. The Dutch Food Guide was used as a guideline for a healthy diet and daily amounts for the different food groups (36). Participants were advised to make small changes in their daily life according to this guideline. No caloric restriction was advised. More information about which CBT techniques were used at each session and information about the daily amounts according to the Dutch Food Guide were described in the study protocol (34). Drop-out is a well-known problem in lifestyle programs, therefore we used an outreach approach to motivate participants to come to the group meetings, unless the participant indicated to withdraw from the study. Participants were called or emailed several times when they were not present during a group-meeting to motivate them to come to the next meeting.

\section{Lifestyle intervention with additional Short Message Service (LS with SMS)}

After 3 months of LS, half of the participants in the LS received additional support by tailored SMS via their mobile phone. Participants sent weekly self-monitored information regarding their diet, physical 
activity and emotions by SMS to the psychologist. Participants received feedback on their messages to provide social support, encourage positive behavior and empower behavioral strategies. Besides, participants received two messages per week addressing eating behavior.

\section{Care as usual (CAU, control group)}

The CAU group had 4 short, unstructured consultations with their treating physician during the standardized screenings at our outpatient clinic at 3, 6, 9 and 12 months. Participants in the CAU group were encouraged to lose weight through publicly available services (i.e. diets, visiting a dietician, going to the gym or participating in public programs such as Weight Watchers $\left.{ }^{\circledR}\right)$. The physician also mentioned the risk of overweight for both mother and child, and the relation between overweight and fertility.

\section{Randomization}

Participants who were assigned to either: 1) 20 CBT lifestyle group sessions including 9 months of electronic feedback through Short Message Service (SMS) via their mobile phone (LS with SMS) 2) 20 CBT lifestyle group sessions (LS without SMS) or 3) to the control group who received usual care (CAU). Written informed consent was obtained from all participants before the study. At baseline, participants were randomized at a 1:1:1 ratio using a computer-generated random numbers table by a research nurse.

\section{Outcomes}

The DEBQ (15) was used to assess eating in response to diffuse emotions (diffuse), eating in response to clearly labeled emotions (emotional eating), eating in response to the sight or smell of food (external eating), and eating less than desired to lose or maintain body weight (dietary restraint). This questionnaire consists of 33 items measuring 4 subscales. The subscale scores range between 1 and 5 , with a higher score reflecting a higher degree of the relevant eating behavior.

The EDEQ $(37,38)$ was used to measure specific eating disorders. This questionnaire consists of 36 items measuring 4 subscales: restraint, shape concerns, weight concerns, eating concerns, and a global score. The subscale scores range between 0 and 6 . A higher score indicates more severe eating psychopathology. A global score or subscale score of 4 or higher is considered clinically significant. In women with PCOS, a mean EDEQ score of 2.38 has been reported compared to 1.29 in the general population (28).

The Beck Depression Inventory (BDI-II) is a validated and widely used questionnaire in depression trials assessing the severity of depressive symptoms over the previous 2 weeks, according to the DSM5 criteria. The BDI-II is a 21 -item self-report questionnaire with items rated on a 4-point scale (0-3) and are summed to give a total score (range $0-63$ ). A higher score on the BDI-II denotes more severe 
depression. Scores of 0-13 indicate minimal depression, 14-19 (mild depression), 20-28 (moderate depression) and 29-63 (severe depression) (39).

\section{Statistical considerations}

The power calculation was based on the primary outcome of the LS intervention: weight $(\mathrm{kg})$. The method described by Aberson (25) was applied, with a power of 0.90, a 2-sided alpha of 0.025 (corrected for the interim analysis as described in the study protocol) and 5 repeated measures linearly decreasing. All variables were analyzed based on the intention-to-treat population, defined as all allocated participants. Multilevel or mixed regression modeling was applied for longitudinal outcomes. Mixed modeling can efficiently deal with missing data and unbalanced time-points $(40,41)$. This means that, additionally, patients without complete follow-ups could be included in the analyses, without imputation. Study group, linear and logarithmic time and interactions were included as independent variables. The deviance statistic (42) using restricted maximum likelihood (43) was applied to determine the covariance structure thus taking into account the situation when e.g. the deviation at baseline is different from the deviations at follow-ups. In the case of a non-normal distribution, a bootstrap procedure with 10,000 samples was performed to obtain a more reliable outcome. The bootstrap mixed model analyses were performed utilizing IBM Corp (Released 2017. IBM SPSS Statistics for Windows, Version 25.0. Armonk, NY: IBM Corp).

To test if weight, depression, androgens, insulin, the homeostatic model assessment for insulin resistance (HOMA-IR) and cortisol mediated the effect of LS on eating behavior, we used multilevel longitudinal mediation or indirect effect analyses. Paths $a, b, t$ and t $\grave{c}$ were estimated employing multilevel regression analyses. Firstly, we determined whether paths $b$ were significant. When path $b$ was not significant, mediation is unlikely. We adjusted the Sobel-Goodman test for the indirect effect of the independent variable on the dependent variable as reported by MacKinnon and Dwyer (44) following the recommendations by Krull and MacKinnon (45) for multilevel mediation analyses. The significance of the mediated effect is given by:

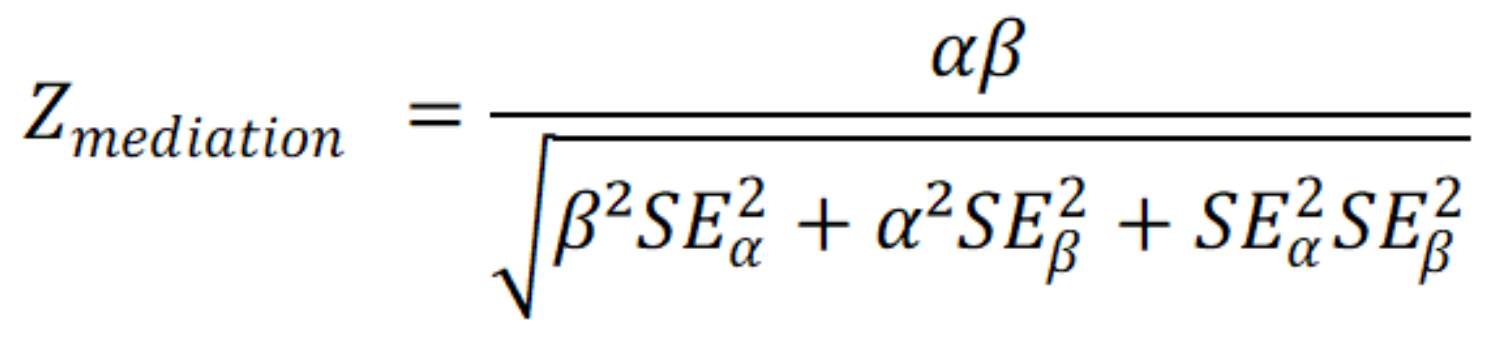

(46).

Cohen's $d$ effect sizes were calculated by dividing the differences between time-point and baseline estimations by the estimated baseline standard deviation. The guidelines of Cohen were used: effect 
sizes of 0.20 were considered as small, 0.50 as medium and 0.80 as large (47). $p$-values $<0.05$ were considered significant.

\section{Results}

Between August $2^{\text {nd }} 2010$ and March 11 $1^{\text {th }} 2016$, all 535 eligible women were asked to participate and 209 provided written informed consent, of whom 26 were included in a pilot study. At baseline, 60 participants were randomized to CAU, 60 to LS with SMS and 63 participants to LS without SMS, resulting in a total of 183. Of these 183 participants, 24 completed CAU, 16 completed LS with SMS and 27 completed LS without SMS. An overall completion rate of 67/183 (36.6\%) was observed, Figure 1. At baseline, 179 participants filled in eating behavior questionnaires. In total, 394 measurements were available for this analyses. The baseline characteristics of the participants are shown in Table 1.

\section{Changes in Disordered eating (EDEQ)}

EDEQ global scores decreased in CAU by $+47.5 \%$ and increased during LS by $-4.2 \%$. The difference between the CAU and LS was significant (Cohen's $d:-0.72 ; p=0.007$ ), Figure 2. No significant difference were observed in EDEQ global scores between LS with SMS compared to LS without SMS (Cohen's d: $0.28 ; P=0.399)$. During the study period, no significant difference was observed for the EDEQ subscale restraint $[A](P=0.254)$ and eating concern $[D](P=0.116)$ between CAU compared to $L S$. Furthermore, the subscale shape concern $[B](P=0.016)$ and weight concern $[C](P=0.007)$ changed significantly between CAU compared to LS, Figure 3. If we compared the difference between between LS without SMS and LS with SMS, no difference were found for the EDEQ global score ( $P=0.399)$, shape concern $(P=0.992)$ weight concern $(P=0.790)$ and eating concern $(P=0.954)$ between these two groups. Only for the subscale restraint we found a significant difference between LS without SMS and LS with SMS $(P=0.015)$. During LS without SMS restrained eating scores remained stable while restrained eating scores worsened in LS with SMS, supplemental Table 1.

\section{Changes in Eating behavior (DEBQ)}

The 4 subscales of the DEBQ for diffuse (Cohen's d: $-0.30 ; P=0.181$ ), emotional (Cohen's $d:-0.18$; $P=0.457$ ), restraint (Cohen's $d:-0.09 ; P=0.761$ ) and external eating (Cohen's $d:-0.10 ; P=0.675$ ) did not change significantly in CAU compared to LS. The same pattern was found if we compared LS without SMS to LS with SMS for diffuse (Cohen's d: $0.04 ; P=0.855$ ), emotional (Cohen's d: $-0.28 ; P=0.296$ ), restraint (Cohen's d: 0.26; $P=0.372$ ) and external eating (Cohen's d: 0.04; $P=0.142$ ), supplemental Table 2.

\section{Mediation of androgens, weight and depression}


A mediating variable $M$ is a variable that lies within the causal chain between an independent variable $X$ and a dependent variable $Y$ and represents the mechanism of change (Figure 4). Panel A indicates a hypothetical causal relationship in which the lifestyle intervention $(X)$ affects eating behavior measured with the EDEQ global score $(Y)$. In Panel $B$, this relationship is hypothesized to be mediated: the lifestyle intervention $(X)$ is hypothesized to reduce weight $(M)$, which in turn would reduce EDEQ global scores $(Y)$. In this way, the effect of the lifestyle intervention on eating behavior should primarily take place through paths $a$ and $b$, rather than through path tch. As a result, we found no mediation in the relationship between lifestyle and EDEQ global scores with weight as a mediator $(P=0.832)$. We also tested 9 other potential mediators (testosterone, androstenedione, dehydro-epiandrosterone (DHEA), insulin, HOMA-IR, cortisol, oligomenorrhea and depression. We found no mediation by these variables in the relationship between lifestyle and EDEQ global scores over time. These results indicate that metabolic features of PCOS, sex steroids, weight and psychological measures were not involved in the observed effects of LS on eating behavior over time.

\section{Table 1}

\section{Baseline characteristics by trial group}




\begin{tabular}{|c|c|c|c|}
\hline & $\begin{array}{l}\text { CAU } \\
(\mathrm{N}=60) \\
\text { Median [IQR] }\end{array}$ & $\begin{array}{l}\text { LS without SMS }(\mathrm{N}=61) \\
\text { Median [IQR] }\end{array}$ & $\begin{array}{l}\text { LS with SMS } \\
(\mathrm{N}=58) \\
\text { Median [IQR] }\end{array}$ \\
\hline EDEQ total & $1.7[0.13-2.7]$ & $1.8[0.0-3.1]$ & $2.1[0.78-2.8]$ \\
\hline Subscale restrained & $1.4[0.0-2.6]$ & $1.2[0.0-2.6]$ & $1.3[0.2-2.9]$ \\
\hline Subscale shape concern & $2.4[0.0-3.4]$ & $2.6[0.0-4.3]$ & $2.8[0.7-3.7]$ \\
\hline Subscale weight concern & $2.1[0.1-3.6]$ & $2.4[0.0-3.8]$ & $2.7[0.8-3.8]$ \\
\hline Subscale eating concern & $0.4[0.0-1.4]$ & $0.2[0.0-1.4]$ & $0.8[0.0-1.8]$ \\
\hline \multicolumn{4}{|l|}{ DEBQ } \\
\hline Subscale diffuse & $2.9[2.0-3.3]$ & $3.3[2.0-3.9]$ & $3.3[2.3-4.0]$ \\
\hline Subscale emotional & $2.3[1.7-2.8]$ & $2.7[1.8-2.7]$ & $2.7[1.9-3.6]$ \\
\hline Subscale restrained & $3.2[2.8-3.6]$ & $3.0[2.7-3.4]$ & $3.1[2.7-3.6]$ \\
\hline Subscale external & $2.7[2.2-3.0]$ & $2.9[2.5-3.3]$ & $2.9[2.4-3.1]$ \\
\hline Age (year) & $28.0[26.0-32.0]$ & $30.0[27.0-33.0]$ & $28.0[26.0-32.0]$ \\
\hline Attempting to conceive (months) & 24 [13.0-61.0] & $24.0[14.0-48.0]$ & $20.0[8.0-31.0]$ \\
\hline Weight (kg) & $84.0[79.0-97.3]$ & $89.0[80.0-104.0]$ & 94.5 [85.0-106.3] \\
\hline BMI $\left(\mathrm{kg} / \mathrm{m}^{2}\right)$ & $30.6[29.3-34.4]$ & $33.5[30.5-36.0]$ & $33.6[31.0-36.8]$ \\
\hline Weight loss (5\%) & $21.8[8.5-45.5]$ & $52.8[23.2-80.5]$ & $85.7[51.3-97.2]$ \\
\hline Weight loss (10\%) & $6.8[1.7-23.5]$ & $12.2[3.2-36.7]$ & $45.9[15.4-79.8]$ \\
\hline \multirow[t]{2}{*}{ Modified Ferriman-Gallwey score } & $3[1-6]$ & $4[2-9]$ & $3[1-9]$ \\
\hline & $\mathrm{N}(\%)$ & $\mathrm{N}(\%)$ & $\mathrm{N}(\%)$ \\
\hline Binge eating episodes & $25(41.7)$ & $36(57.1)$ & $25(41.7)$ \\
\hline \multicolumn{4}{|l|}{ Menstrual cycle } \\
\hline Oligomenorrhea & $51(85.0)$ & $52(85.2)$ & 37 (63.8) \\
\hline Amenorrhea & $6(10.0)$ & 7 (11.5) & 17 (29.3) \\
\hline Regular & $3(5.0)$ & $2(3.3)$ & $2(3.4)$ \\
\hline Caucasian & $19(32.2)$ & $20(32.8)$ & $26(44.8)$ \\
\hline \multicolumn{4}{|l|}{ Education } \\
\hline Low & $7(11.7)$ & $4(6.6)$ & $5(8.6)$ \\
\hline
\end{tabular}


Note: IQR= Interquartile range, $\mathrm{CAU}=\mathrm{Care}$ as Usual, LS without $\mathrm{SMS}=$ Lifestyle without Short Message Service, LS with SMS= Lifestyle with Short Message Service, EDEQ= Eating Disorder Examination Questionnaire, DEBQ= Dutch Eating Behavior Questionnaire

\section{Discussion}

To the best of our knowledge, we performed one of largest RCT investigating weight loss during a threecomponent CBT LS intervention in women with PCOS. Several one- or two component interventions achieved short-term weight loss in women with PCOS (48) and did not examine eating behavior. We thus performed an analysis of the eating behavior data that was collected in the RCT. In accordance with our hypothesis, disordered eating behavior improved during a three-component LS program which combined nutritional advice, exercise and cognitive behavioral therapy while women in CAU developed more disordered eating behavior. We found a medium to large effect size (expressed in Cohen's D) for the changes in eating behavior if we compared the effects of LS to CAU. This suggest that the lifestyle intervention is more effective than CAU to change eating behavior in women with PCOS.

Many LS interventions in women with PCOS encouraged dietary restraint by describing a very strict diet with a calorie deficit of 500 to 1000 calories per day (49-51). It is no surprise that women lost between 4.4 and 8.9 kilograms during the study period due to the amount of calories they were allowed to consume. During these diets, participants temporarily restricted their food intake and did not change their behavior which is necessary for long-term weight loss (52). In the general population dieting is the strongest risk factor to develop disordered eating $(53,54)$. Many women with PCOS have tried several diets and often report that weight loss is more challenging for them (30). The combination of these two factors may place them at risk for disordered eating behavior (53). During our three-component LS intervention women lost weight (35) while disordered eating behavior improved. Suggesting that the combination of the three-components is also effective for changes in eating behavior. As described in the protocol paper (34), all sessions underlined the development of a personal healthy diet that could be sustained for a longer period of time. Women were advised to make small changes in their current diet based on the daily amounts of the Dutch Food guide without restricting or counting calories for example eating more bread or less meat. During the CBT sessions, much time was spend on realistic goal setting for weight loss, self-monitoring of food intake, the development of alternative behaviors, the sight and smell of food, social eating situations and cognitive restructuring by using thought records. The intervention was designed to develop specific cognitive and behavioral strategies in order to develop different eating behavior necessary for weight loss. All three components interact with each other and therefore it is unclear which component contributed the most to the changes in eating behavior.

Within the general population, risk factors for disordered eating were associated with psychosocial, demographic, environmental and genetic factors (55-57). It is unclear why so many women with PCOS 
have disordered eating behaviors. The current literature suggests that distress, low self-esteem (58) and depression (29) were associated with disordered eating in women with PCOS. This is in line with the general population where higher depression scores were related to eating disorders (59). Therefore, we tested different mediators in the relationship between lifestyle treatment and changes in disordered eating behavior. Surprisingly, we found no significant mediation by weight or depression scores that could explain the changes in eating behavior during the lifestyle treatment. We also tested other potential mediators since a relationship between high levels of androgens and binge eating was found in the general population (60). In women with PCOS, a connection between high androgen levels, polycystic ovaries and behavioral deficits such as impulsivity was suggested, which could make women with PCOS more vulnerable for bulimia nervosa (61). Others also suggested that the irregular menstrual cycle (oligomenorrhea) $(62,63)$ or high levels of insulin $(27)$ may lead to increased hunger and psychological distress, which could result in more binge eating. As a result, we also found no mediation by weight, depression, androgens, insulin, HOMA-IR, cortisol or oligomenorrhea. This could suggest that the lifestyle intervention itself and not depression, weight or androgens were involved in the changes in eating behavior that were observed during the lifestyle intervention.

A limitation of the present trial is that we observed high drop-outs rates comparable to other obesity treatments in the general population $(64,65)$. In lifestyle programs designed for women with PCOS dropout rates of around $25 \%$ were reported. It is still unclear if patient or intervention related factors are related to drop-out (66). Drop-outs could effected the results of many lifestyle interventions because outcomes were most of the times based on complete cases analyses. Complete cases analyses can overestimate weight loss because study completers achieve more weight loss than drop-outs (67). To prevent these overestimations, we have chosen a statistical method that included all available data even if participants dropped out during the study period. Despite an overall drop-out rate of $63.4 \%$, the mixed multilevel model was based on a high number of measurements (394 in total) belonging to 183 participants.

Future research should examine whether women with PCOS and different types of eating behavior benefit from surgical or nonsurgical weight loss interventions. Each kind of eating behavior has its etiology, and requires a different treatment (19). At the moment, we are performing a new RCT to test the effects of gastric bypass surgery versus the current three-component lifestyle intervention in women with PCOS. Especially to examine which treatment works best for this large and diverse group of women.

\section{Conclusion}

Treatment by a three-component lifestyle program that combined nutritional advice, exercise and CBT resulted in a medium to large effect size and significant improvements in disordered eating behavior compared to CAU. Neither weight loss, depression, testosterone, androstenedione, DHEA, insulin, HOMA-IR nor cortisol did mediate this effect. A multidisciplinary lifestyle treatment is effective to improve disordered eating behavior in women with PCOS.

\section{Declarations}




\section{Ethics approval and consent to participate:}

This study was approved by the Medical Research Ethics Committee of the Erasmus MC in Rotterdam; reference number MEC 2008-337

\section{Consent for publication:}

Not applicable

\section{Availability of data and materials:}

The datasets used and/or analysed during the current study are available from the corresponding author on reasonable request.

\section{Competing interests:}

GJ, $A D L, R T, A B$, and JB have nothing to declare. JL has received unrestricted research grants from Ansh Labs, Ferring and Roche Diagnostics. He received consultancy fees from the following companies: Ansh Labs, Ferring, Roche Diagnostics, and Titus Healthcare.

\section{Funding:}

The study was supported by an institutional grant for a PhD position.

\section{Authors' contributions:}

GJ, ADL, RT, AB, JL and JB and made substantial contributions to the conceptualization, study design, execution, implementation and writing of this paper. GJ and RT performed the formal statistical analyses.

\section{Acknowledgements:}

We thank the entire PCOS team of the Erasmus MC.

\section{Authors' information (optional):}

Geranne Jiskoot works as a psychologist and obtained a master degree in epidemiology in 2014. She is a PhD Candidate at the Division of Reproductive Endocrinology and Infertility and at the Department of Medical Psychology and Psychotherapy of the Erasmus MC. Her main research interests include lifestyle 
treatment in women with Polycystic Ovary Syndrome (PCOS) and the emotional aspects of women dealing with premature ovarian insufficiency (POI).

\section{References}

1. March WA, Moore VM, Willson KJ, Phillips DI, Norman RJ, Davies MJ. The prevalence of polycystic ovary syndrome in a community sample assessed under contrasting diagnostic criteria. Journal of Human Reproduction. 2010;25(2):544-51.

2. Bozdag G, Mumusoglu S, Zengin D, Karabulut E, Yildiz BO. The prevalence and phenotypic features of polycystic ovary syndrome: a systematic review and meta-analysis. Human Reproduction. 2016;31(12):2841-55.

3. Azziz R, Carmina E, Chen Z, Dunaif A, Laven JS, Legro RS, et al. Polycystic ovary syndrome. Nature Reviews Disease Primers. 2016;2:16057.

4. Lim SS, Davies MJ, Norman RJ, Moran LJ. Overweight, obesity and central obesity in women with polycystic ovary syndrome: a systematic review and meta-analysis. Human Reproduction Update. 2012;18(6):618-37.

5. Laven JS, Imani B, Eijkemans MJ, Fauser BC. New approach to polycystic ovary syndrome and other forms of anovulatory infertility. Obstetrical and Gynecological Survey. 2002;57(11):755-67.

6. Teede, Moran L. Polycystic ovary syndrome: a complex condition with psychological, reproductive and metabolic manifestations that impacts on health across the lifespan. BMC Medicine. 2010;8:41.

7. Meun C, Franco OH, Dhana K, Jaspers L, Muka T, Louwers Y, et al. High androgens in postmenopausal women and the risk for atherosclerosis and cardiovascular disease: the Rotterdam Study. J Clin Endocrinol Metab. 2018.

8. Veltman-Verhulst SM, Boivin J, Eijkemans MJ, Fauser BJ. Emotional distress is a common risk in women with polycystic ovary syndrome: a systematic review and meta-analysis of 28 studies. Human Reproduction Update. 2012;18(6):638-51.

9. Cooney, Lee, Sammel, Dokras. High prevalence of moderate and severe depressive and anxiety symptoms in polycystic ovary syndrome: a systematic review and meta-analysis. Human Reproduction. 2017;32(5):1075-91.

10. Teede, Deeks A, Moran L. Polycystic ovary syndrome: a complex condition with psychological, reproductive and metabolic manifestations that impacts on health across the lifespan. BMC Medicine. 2010;8:41.

11. Teede H, Deeks A, Moran L. Polycystic ovary syndrome: a complex condition with psychological, reproductive and metabolic manifestations that impacts on health across the lifespan. BMC Med. 2010;8:41.

12. American Psychiatric A. Diagnostic and statistical manual of mental disorders (DSM-5®): American Psychiatric Pub; 2013. 
13. Fairburn CG, Bohn K. Eating disorder NOS (EDNOS): an example of the troublesome "not otherwise specified"(NOS) category in DSM-IV. Behaviour research and therapy. 2005;43(6):691-701.

14. American Psychiatric A. American Psychiatric Association Practice Guidelines for the treatment of psychiatric disorders: compendium 2006: American Psychiatric Pub; 2006.

15. Van Strien T, Frijters JER, Bergers GPA, Defares PB. The Dutch Eating Behavior Questionnaire (DEBQ) for assessment of restrained, emotional, and external eating behavior. International journal of eating disorders. 1986;5(2):295-315.

16. van Strien T, Cebolla A, Etchemendy E, Gutierrez-Maldonado J, Ferrer-Garcia M, Botella C, et al. Emotional eating and food intake after sadness and joy. Appetite. 2013;66:20-5.

17. Van Strien T, Herman CP, Verheijden MW. Eating style, overeating, and overweight in a representative Dutch sample. Does external eating play a role? Appetite. 2009;52(2):380-7.

18. Polivy J, Herman PC. Restrained eating. Obesity. 1980:208-25.

19. van Strien T. Causes of emotional eating and matched treatment of obesity. Current diabetes reports. 2018;18(6):35.

20. Lowe MR, Thomas JG. Measures of restrained eating: Conceptual evolution and psychometric update. Handbook of assessment methods for obesity and eating behaviors. 2009:137-85.

21. Stroebe W. Restrained eating and the breakdown of self-regulation. 2008.

22. Langeveld $\mathrm{M}$, DeVries $\mathrm{JH}$. The long-term effect of energy restricted diets for treating obesity. Obesity. 2015;23(8):1529-38.

23. Bohrer BK, Forbush KT, Hunt TK. Are common measures of dietary restraint and disinhibited eating reliable and valid in obese persons? Appetite. 2015;87:344-51.

24. Werrij MQ, Jansen A, Mulkens S, Elgersma HJ, Ament AJ, Hospers HJ. Adding cognitive therapy to dietetic treatment is associated with less relapse in obesity. Journal of Psychosomatic Research 2009;67(4):315-24.

25. Linardon J, Wade TD, de la Piedad Garcia X, Brennan L. The efficacy of cognitive-behavioral therapy for eating disorders: A systematic review and meta-analysis. J Consult Clin Psychol. 2017;85(11):1080-94.

26. Thannickal A, Brutocao C, Alsawas M, Morrow A, Zaiem F, Murad MH, et al. Eating, Sleeping and Sexual Function Disorders in Women with Polycystic Ovary Syndrome (PCOS): a systematic review and meta-analysis. Clin Endocrinol (Oxf). 2020.

27. Jeanes YM, Reeves S, Gibson EL, Piggott C, May VA, Hart KH. Binge eating behaviours and food cravings in women with Polycystic Ovary Syndrome. Appetite. 2017;109:24-32.

28. Lee I, Cooney LG, Saini S, Smith ME, Sammel MD, Allison KC, et al. Increased risk of disordered eating in polycystic ovary syndrome. Fertil Steril. 2017;107(3):796-802.

29. Greenwood EA, Pasch LA, Cedars MI, Huddleston HG. Obesity and depression are risk factors for future eating disorder-related attitudes and behaviors in women with polycystic ovary syndrome. Fertility and Sterility. 2020;113(5):1039-49. 
30. Larsson I, Hulthen L, Landen M, Palsson E, Janson P, Stener-Victorin E. Dietary intake, resting energy expenditure, and eating behavior in women with and without polycystic ovary syndrome. Clin Nutr. $2016 ; 35(1): 213-8$.

31. Teede, Misso ML, Costello MF, Dokras A, Laven J, Moran L, et al. Recommendations from the international evidence-based guideline for the assessment and management of polycystic ovary syndrome. Fertility and Sterility. 2018;110(3):364-79.

32. Dalle Grave R, Calugi S, Centis E, Marzocchi R, El Ghoch M, Marchesini G. Lifestyle modification in the management of the metabolic syndrome: achievements and challenges. Diabetes, metabolic syndrome and obesity: targets and therapy. 2010;3:373.

33. Castelnuovo G, Pietrabissa G, Manzoni GM, Cattivelli R, Rossi A, Novelli M, et al. Cognitive behavioral therapy to aid weight loss in obese patients: current perspectives. Psychol Res Behav Manag. 2017; 10:165-73.

34. Jiskoot G, Benneheij SH, Beerthuizen A, de Niet JE, de Klerk C, Timman R, et al. A three-component cognitive behavioural lifestyle program for preconceptional weight-loss in women with polycystic ovary syndrome (PCOS): a protocol for a randomized controlled trial. Reprod Health. 2017;14(1):34.

35. Jiskoot G, Dietz de Loos A, Beerthuizen A, Timman R, Busschbach J, Laven J. Weight reduction through a cognitive behavioral therapy lifestyle intervention in polycystic ovary syndrome (PCOS): the primary outcome of a randomized controlled trial. Obesity. 2020;in press.

36. Brink E, van Rossum C, Postma-Smeets A, Stafleu A, Wolvers D, van Dooren C, et al. Development of healthy and sustainable food-based dietary guidelines for the Netherlands. Public health nutrition. 2019;22(13):2419-35.

37. Fairburn CG, Beglin SJ. Assessment of eating disorders: interview or self-report questionnaire? Int J Eat Disord. 1994;16(4):363-70.

38. Fairburn CG, Beglin SJ. Eating disorder examination questionnaire. Cognitive behaviour therapy and eating disorders. 2008;309:313.

39. Beck AT, Steer RA, Ball R, Ranieri W. Comparison of Beck Depression Inventories -IA and -II in psychiatric outpatients. J Pers Assess. 1996;67(3):588-97.

40. Roderick JAL, Donald BR. Statistical analysis with missing data: John Wiley \\&amp; Sons, Inc.; 1986.

41. Little R, Rubin D. Statistical analysis with missing data. New York: John Wiley and Sons. 1987.

42. Singer JD, Willett JB. Applied longitudinal data analysis: Modeling change and event occurrence: Oxford university press; 2003.

43. Verbeke G, Molenberghs G. Inference for the marginal model. Linear Mixed Models for Longitudinal Data. 2000:55-76.

44. MacKinnon DP, Dwyer JH. Estimating mediated effects in prevention studies. Evaluation review. 1993;17(2):144-58.

45. Krull JL, MacKinnon DP. Multilevel mediation modeling in group-based intervention studies. Evaluation review. 1999;23(4):418-44. 
46. Mathieu JE, Taylor SR. Clarifying conditions and decision points for mediational type inferences in organizational behavior. Journal of Organizational Behavior: The International Journal of Industrial, Occupational and Organizational Psychology and Behavior. 2006;27(8):1031-56.

47. Cohen J. A power primer. Psychological bulletin. 1992;112(1):155.

48. Moran, Hutchison SK, Norman RJ, Teede HJ. Lifestyle changes in women with polycystic ovary syndrome. Cochrane Database of Systematic Reviews. 2011(7).

49. Hoeger KM, Kochman L, Wixom N, Craig K, Miller RK, Guzick DS. A randomized, 48-week, placebocontrolled trial of intensive lifestyle modification and/or metformin therapy in overweight women with polycystic ovary syndrome: a pilot study. Fertil Steril. 2004;82(2):421-9.

50. Palomba S, Giallauria F, Falbo A, Russo T, Oppedisano R, Tolino A, et al. Structured exercise training programme versus hypocaloric hyperproteic diet in obese polycystic ovary syndrome patients with anovulatory infertility: a 24-week pilot study. Hum Reprod. 2008;23(3):642-50.

51. Mutsaerts MA, van Oers AM, Groen H, Burggraaff JM, Kuchenbecker WK, Perquin DA, et al. Randomized Trial of a Lifestyle Program in Obese Infertile Women. N Engl J Med. 2016;374(20):1942-53.

52. Wing RR, Phelan S. Long-term weight loss maintenance. Am J Clin Nutr. 2005;82(1 Suppl):222S-5S.

53. Watson HER, Dreher C, Steele A. Eating disorders prevention, treatment \& management: An evidence review. The National Eating Disorders Collaboration. 2010.

54. Watson $\mathrm{H}$. Evaluating the risk of harm of weight-related public messages. National Eating Disorders Collaboration. 2011.

55. Klump KL, Suisman JL, Burt SA, McGue M, lacono WG. Genetic and environmental influences on disordered eating: An adoption study. J Abnorm Psychol. 2009;118(4):797-805.

56. Klump KL, Burt SA, McGue M, lacono WG. Changes in genetic and environmental influences on disordered eating across adolescence: a longitudinal twin study. Arch Gen Psychiatry. 2007;64(12):1409-15.

57. Neumark-Sztainer D, Wall M, Story M, Sherwood NE. Five-year longitudinal predictive factors for disordered eating in a population-based sample of overweight adolescents: implications for prevention and treatment. Int J Eat Disord. 2009;42(7):664-72.

58. Tay CT, Teede HJ, Hill B, Loxton D, Joham AE. Increased prevalence of eating disorders, low selfesteem, and psychological distress in women with polycystic ovary syndrome: a community-based cohort study. Fertil Steril. 2019;112(2):353-61.

59. Hudson JI, Hiripi E, Pope HG, Jr., Kessler RC. The prevalence and correlates of eating disorders in the National Comorbidity Survey Replication. Biol Psychiatry. 2007;61(3):348-58.

60. Baker JH, Girdler SS, Bulik CM. The role of reproductive hormones in the development and maintenance of eating disorders. Expert review of obstetrics \& gynecology. 2012;7(6):573-83.

61. Sundblad C, Bergman L, Eriksson E. High levels of free testosterone in women with bulimia nervosa. Acta Psychiatrica Scandinavica. 1994;90(5):397-8. 
62. Paganini C, Peterson G, Stavropoulos V, Krug I. The overlap between binge eating behaviors and polycystic ovarian syndrome: An etiological integrative model. Current pharmaceutical design. 2018;24(9):999-1006.

63. Ålgars M, Huang L, Von Holle AF, Peat CM, Thornton LM, Lichtenstein P, et al. Binge eating and menstrual dysfunction. Journal of psychosomatic research. 2014;76(1):19-22.

64. Lantz H, Peltonen M, Ågren L, Torgerson JS. A dietary and behavioural programme for the treatment of obesity. A 4-year clinical trial and a long-term posttreatment follow-up. Journal of internal medicine. 2003;254(3):272-9.

65. Ortner Hadžiabdić M, Mucalo I, Hrabač P, Matić T, Rahelić D, Božikov V. Factors predictive of drop-out and weight loss success in weight management of obese patients. Journal of human nutrition and dietetics. 2015;28:24-32.

66. Mutsaerts M, Kuchenbecker W, Mol B, Land J, Hoek A. Dropout is a problem in lifestyle intervention programs for overweight and obese infertile women: a systematic review. Hum Reprod. 2013;28(4):979-86.

67. Douketis JD, Macie C, Thabane L, Williamson DF. Systematic review of long-term weight loss studies in obese adults: clinical significance and applicability to clinical practice. International journal of obesity. 2005;29(10):1153-67.

\section{Figures}




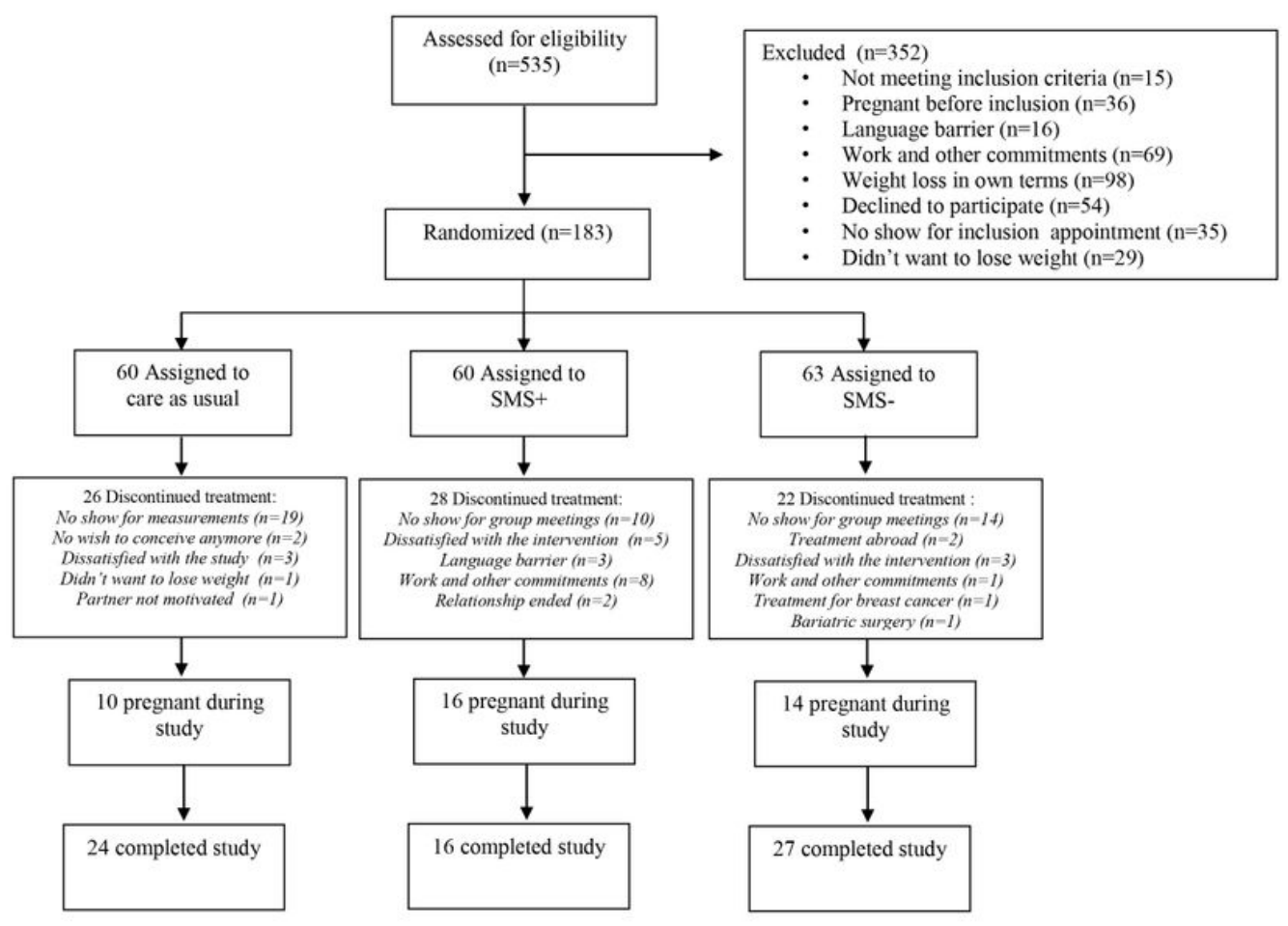

Figure 1

Please See image above for figure legend. 


\section{EDEQ global scores}

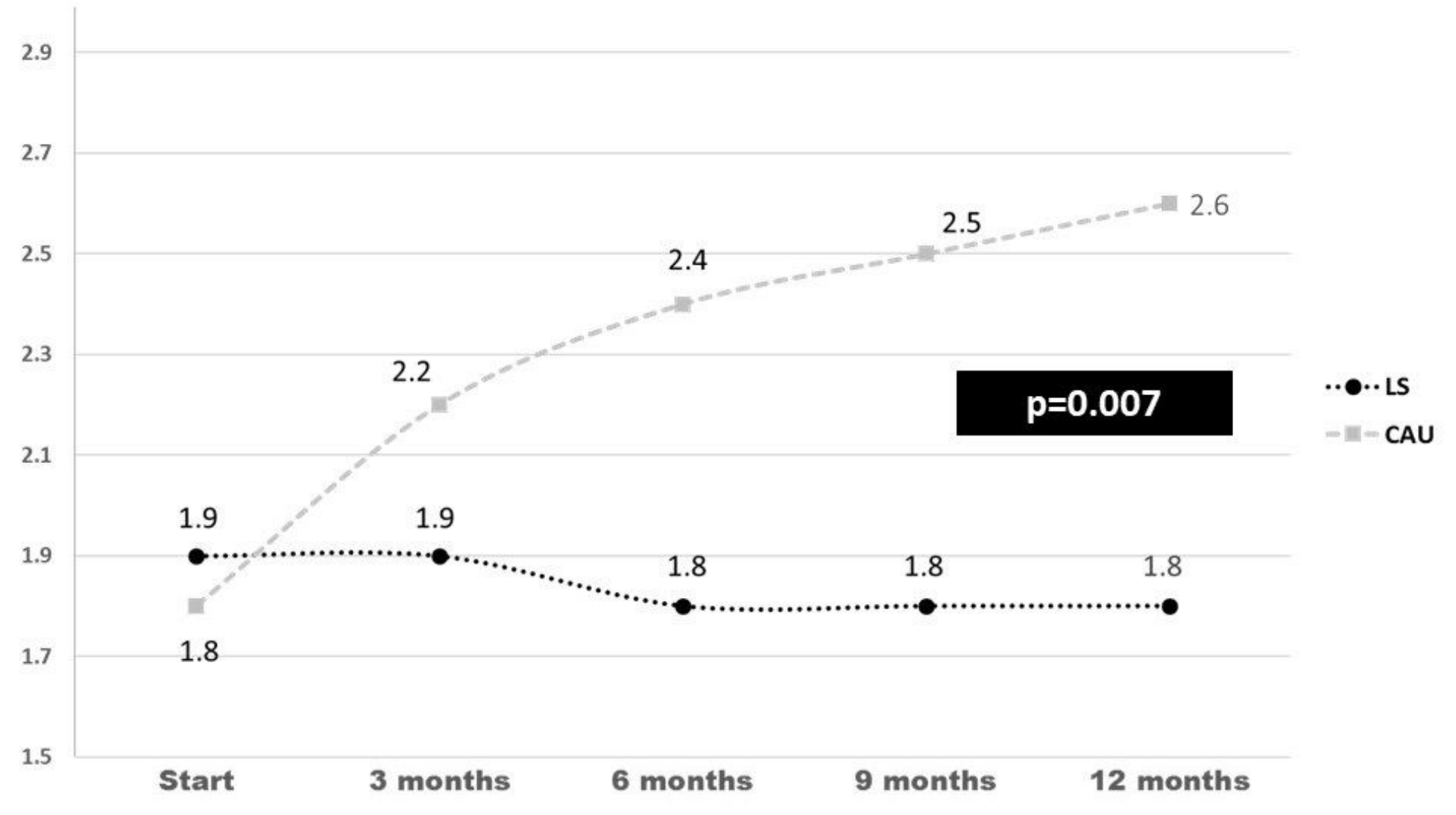

Figure 2

Legend not included with this version 


\section{[A] EDEQ restraint}

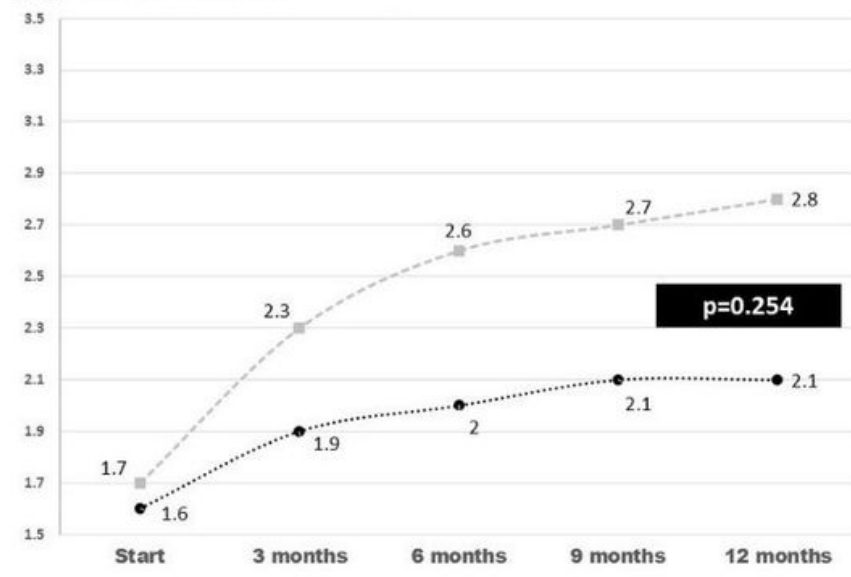

\section{[C] EDEQ weight concern}

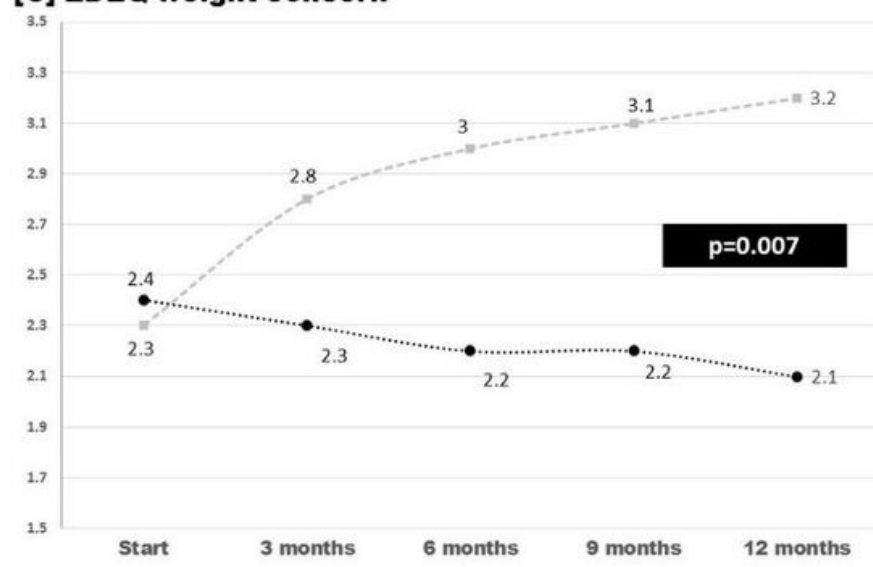

[B] EDEQ shape concern

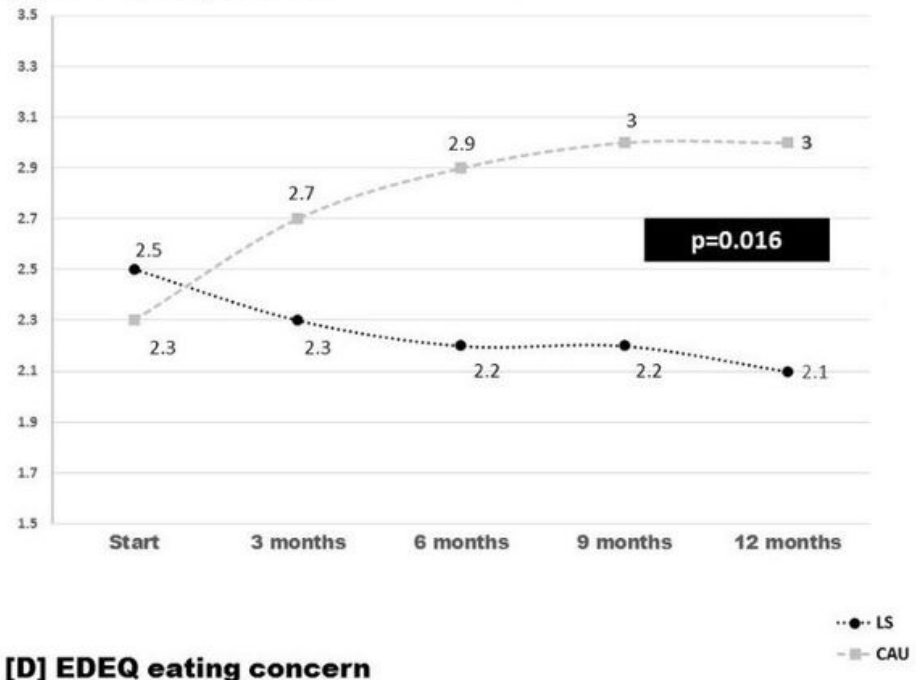

[D] EDEQ eating concern

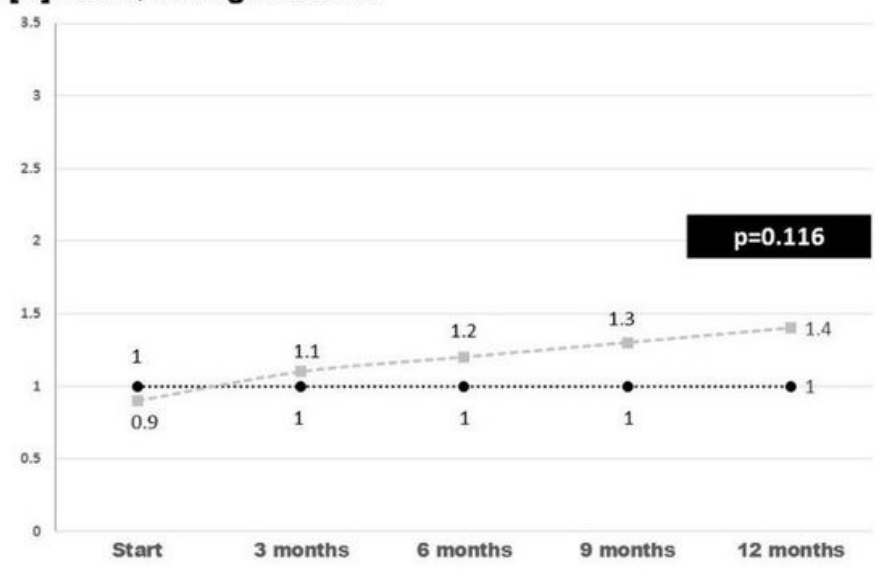

\section{Figure 3}

Legend not included with this version 

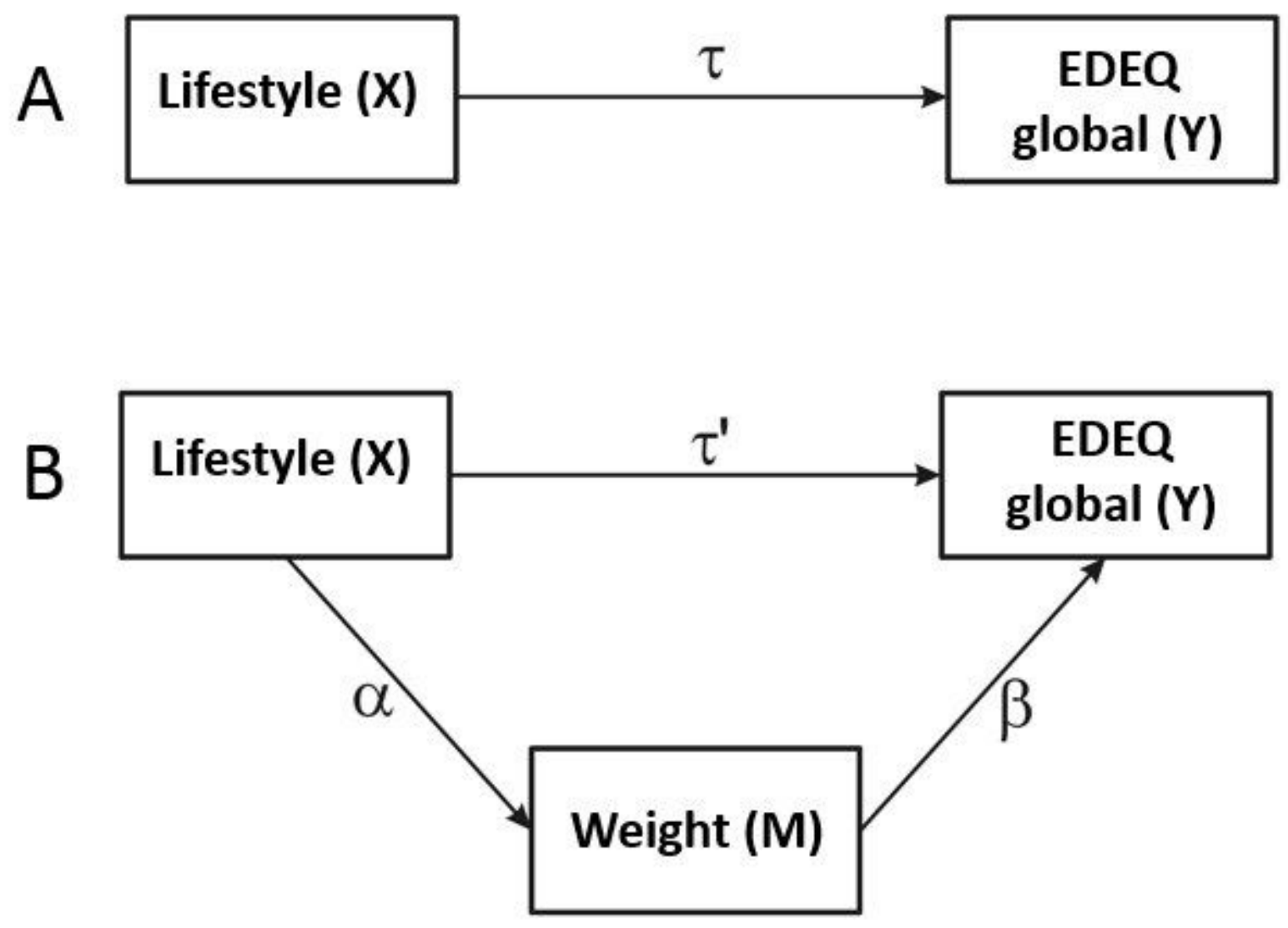

Figure 4

Legend not included with this version

\section{Supplementary Files}

This is a list of supplementary files associated with this preprint. Click to download.

- SupplementalTable1.doc

- SupplementalTable2.doc 